\title{
Early Pseudodepression Anticipating the Appearance of A Frontal Lobe Brain Tumor: Could it Be Considered A Risk Factor? Literature Review and Case Description
}

\author{
Domenico Chirchiglia*, Carmelino Angelo Stroscio, Dorotea Pugliese, Donatella Gabriele, Giusy \\ Guzzi, Giorgio Volpentesta, Angelo Lavano
}

University of Catanzaro, Department of Neurosurgery, Campus Germaneto, Catanzaro, Italy

"Corresponding Author: Dr. Domenico Chirchiglia, University of Catanzaro, Department of Neurosurgery, Campus Germaneto, Vle Europa, 88100, Catanzaro, Italy, Tel: +3909613697410; E-mail: chirchiglia@ unicz.it

Received: 02 October 2018; Accepted: 10 October 2018; Published: 15 October 2018

\begin{abstract}
Background: Frontal lobe brain tumors often cause psychiatric disorders such as depression, if damage is related to dorsolateral part of the prefrontal cortex.It is more appropriate the term pseudodepression in cases where it is secondary to organic causes, such as in brain tumors. Vice versa, it is a very rare condition in which depressive symptoms anticipate the onset of a brain tumor, as showed by few cases reported in literature.
\end{abstract}

Procedure: We describe a case of pseudodepression syndrome followed 20 years after, by the appearance of a frontal lobe meningioma.

Conclusions: There are many hypotheses whereby a depression can anticipate the onset of a brain tumor. According to some authors, depression could be a risk factor for the onset of brain tumors. Hence the need, in a subject suffering from depression, to perform periodic control neuroimaging exams, to detect the possible presence of a brain tumor.

Keywords: Pseudodepression; Brain tumor; Frontal lobe

\section{Introduction}

Depression is a mood disorder characterized by frequent and intense states of dissatisfaction and sadness and loss of pleasure in common daily activities. People suffering from depression live in a condition of constant mood disorder, 
with negative thoughts about themselves and their own future. The most affected age range is between 30 and 50 years [1]. The depressive disorder is about twice as frequent among women. Over the last few years the prevalence of depression has increased steadily and at the same time the age of onset has decreased. At present, depression is considered by the World Health Organization the second cause of disability between physical and psychological illnesses, second only to heart attack [1].

The most common symptoms of depression are loss of energy, sense of fatigue, difficulty in concentration and memory, motor agitation and nervousness, loss or weight gain, sleep disorders (insomnia or hypersomnia), lack of sexual desire and pain. These are the physical symptoms. Cognitive-emotional symptoms are sadness, anguish, despair, sense of helplessness, loss of hope, difficulty in making decisions and solving problems, self-devaluation, pessimistic thinking. In the most severe cases there may be social isolation until suicide attempts [1].

Primary depression involves genetic predisposition and familiarity. Pseudodepression is the form secondary to organic diseases, including brain tumors. Pseudodepression syndrome is typical of frontal tumors, of the dorsolateral part of the prefrontal cortex [1]. We describe the case of pseudodepression, arising 20 years before the discovery of a frontal lobe brain tumor.

\section{Case Report}

The case concerns a 45 year-old-male, suffering from depression, from the age of 25 years. Familiarity with depression was reported (the mother was affected) The symptoms consisted of reduced mood, lack of concentration, fatigue, crying, tendency to be alone, sadness. He was administered over time Hamilton depression rating scale (HDRS). HDRS is based on the 17-item scale.Scores from 0-7 are considered normal, 8-16 mild depression, 17-23 moderate depression while scores over 24 indicate severe depression. HDRS showed almost always a score between 18 and 21 , suggesting a moderate depression. He assumed several antidepressants over the years, achieving inconstant results. He performed brain CT and a brain MRI when he had the first depressive symptoms. Both exams were normal. He continued therapy for about 20 years, obtaining satisfactory results in some periods, less in others, and often changing antidepressant drugs. Paroxetine was demonstrating the most effective drug. For the onset of frontal headache, the patient underwent to a brain MRI control, which showed the presence of a right frontal meningioma. The patient underwent surgical treatment, followed by strong improvement of depression symptoms.

\section{Discussion}

Prevalence of depression among brain tumors is variable, Huang et al. [2] in a recent study found a percentage about $21.7 \%$. Liu et al. [3] found a prevalence of $38 \%$. Pranckeviciene A found that $78 \%$ of 530 patients with brain tumors had psychiatric symptoms, and $18 \%$ of the 530 had only these symptoms as the first clinical manifestation of a brain tumor [4]. Depression can present in different phases (before, during or after diagnosis) of brain tumors. It is known that depression is more commonly in frontal lobe brain tumors, in particular in left frontal lobe tumors. Symptoms may be vague, such as apathy or personality changes. Psychiatric symptoms may be the only presenting symptoms of a brain tumor. These symptoms tend not to be localized to specific anatomical regions. Tumors also cause pressure, edema, and alteration of brain connections Thus, psychiatric symptoms generally have no localizing value. Prefrontal cortex include dorsolateral tumors, causing pseudodepression syndrome, orbitofrontal tumors causing 
disinhibition and personality changes, and ventromedial tumors involved in processing of risk and fear, decision making, self control and morality evaluation.

Pseudodepression syndrome or Kleist's syndrome is an apathetic syndrome, frequent in prefrontal dorsolateral tumors and characterized by negative phenomena, in which the patient loses all forms of interest in himself and the surrounding environment, as well as the initiative and concentration [5]. Usually, the tumor causes depression, in this case depression precedes the tumor by 20 years. Moreover, it is known that brain meningiomas are slowgrowing tumors, often lasting many years. There are studies in literature that show how depression can be a risk factor in developing tumors, especially if there is familiarity with tumors in the patient's clinical history. The literature offers very few cases in which depression is followed by the discovery of a brain tumor. Maruyama et al. [6] reported a case of thyroid carcinoma with solitary cerebral metastasis. The patient had been longer suffering from depression, undergoing years later to neuroimaging examinations, discovering metastasis. Rigau et al. [7] define early depression as high risk factor in developing tumors, especially if it is present inheritance. Gunzel in a study of 365 brain tumor patients found 49 of them who had in clinical history only depression, before the tumor was discovered, correlating psychiatric disorders with the growth of a brain tumor [8]. One hundred and fifty-nine operated patients for new diagnosed brain tumors completed a questionnaire, a single-item 11-point visual analogue scale for measuring psychological distress.Percentage of patients suffering from relevant distress was $48.4 \%$ with scores associated with depression and anxiety.

Prevalence of depression is high shortly after primary neurosurgical treatment in patients with brain tumors and cannot be predicted by objective data. Sources of depression can and should be routinely assessed in these patients overtime [9]. Another study regarding a series of brain tumors showed about $28 \%$ of cases reporting clinically relevant signs of depression [10].

\section{Conclusion}

In short, the studies reported so far have shown a rare correlation between depression and brain tumors, the depression that occurs first and not vice versa. The reasons are not clear.What assumptions can be made? An anticipatory depressive state of a brain tumour is not a frequent condition, with few cases reported in the literature. However, depression as a risk factor for the onset of a brain tumor is a proven fact. It would be useful, in this regard, in situations such as these, to perform control neuroimaging examinations over time, in order to anticipate the eventual discovery of a brain tumor.

\section{Conflict of Interest}

The authors declare no conflict of interest, observation for ethical rules.

\section{References}

1. Ozdilek B, Midi L. Brain tumor presenting with psychiatric symptoms. J Neuropsychiatry Clin Neurosci 23 (2011): E43-E44.

2. Huang J, Zeng C, Xiao J, et al. Association between depression and brain tumor: a systematic review and meta-analysis. Oncotarget 8 (2017): 94932-94943. 
3. Liu W, Xue J, Yu S, et al. Primary central nervous system lymphoma mimicking recurrent depressive disorder: A case report. Oncol Lett 9 (2015): 1819-1821.

4. Pranckeviciene A, Bunevicius A. Depression screening in patients with brain tumors: a review. CNS Oncol 4 (2015): 71-78.

5. Neumärker KJ, Bartsch AJ. 11 Karl Kleist (1879-1960)- a pioneer of neuropsychiatry. Hist Psychiatry 14 (2003): 411-458.

6. Maruyama M, Kobayashi S, Shingu K, et al. Solitary brain metastasis from papillary thyroid carcinoma in a patient with depression: report of a case. Surg Today 30 (2000): 827-830.

7. Rigau V. French brain tumor database: 5-year histological results on 25756 cases. Brain Pathol 21 (2011): 633-644.

8. Günzel H, Tennstedt A. Psychiatric disorders and growth of brain tumors].Psychiatr Neurol Med Psychol (Leipz) 35 (1983): 334-340.

9. Lammens CR, Bleiker EM, Verhoef S, et al. Distress in partners of individuals diagnosed with or at high risk of developing tumors due to rare hereditary cancer syndromes. Psychooncology 20 (2011): 631-638.

10. Fulton JD, Duncan G, Caird FI. Psychiatric presentation of intracranial tumour in the elderly. Int J Geriatr Psychiatry 7 (1992): 411-418.

Citation: Domenico Chirchiglia, Carmelino Angelo Stroscio, Dorotea Pugliese, Donatella Gabriele, Giusy Guzzi, Giorgio Volpentesta, Angelo Lavano. Early Pseudodepression Anticipating the Appearance of A Frontal Lobe Brain Tumor: Could it Be Considered A Risk Factor? Literature Review and Case Description. Journal of Psychiatry and Psychiatric Disorders 2 (2018): 118-121.

\footnotetext{
() $\stackrel{(1)}{\mathrm{N}}$

This article is an open access article distributed under the terms and conditions of the Creative Commons Attribution (CC-BY) license 4.0
} 